\title{
Quantitatively probing two-electron entanglement with a spintronic quantum eraser
}

\author{
Wei Chen, ${ }^{1,2}$ R. Shen, ${ }^{1, *}$ Z. D. Wang, ${ }^{2, \dagger}$ L. Sheng, ${ }^{1}$ B. G. Wang, ${ }^{1,3}$ and D. Y. Xing ${ }^{1}$ \\ ${ }^{1}$ National Laboratory of Solid State Microstructures and Department of Physics, Nanjing University, Nanjing 210093, China \\ ${ }^{2}$ Department of Physics and Center of Theoretical and Computational Physics, The University of Hong Kong, \\ Pokfulam Road, Hong Kong, China \\ ${ }^{3}$ National Center of Microstructures and Quantum Manipulation, Nanjing University, Nanjing 210093, China
}

(Received 26 July 2012; revised manuscript received 7 March 2013; published 16 April 2013)

\begin{abstract}
We design a spintronic quantum eraser to quantitatively probe the two-electron entanglement. It is shown that the concurrence of two spin-entangled electrons is directly given by the Aharonov-Bohm oscillation amplitude of the Fano factor, a measurable current-current correlation, making it rather promising to experimentally quantify the two-electron entanglement. The singlet and triplet entangled states are distinguished by the opposite signs in the Fano factor. Since the main building blocks in the designed setup, an electronic Mach-Zehnder interferometer and a spin filter, have already been implemented, our proposal is particularly pertinent to experiments.
\end{abstract}

DOI: 10.1103/PhysRevB.87.155308

PACS number(s): 85.75.-d, 03.67.Mn, 72.70.+m, 73.23.-b

\section{INTRODUCTION}

Entanglement of electron pairs in solids is a key resource for large-scale implementation of quantum information and computation schemes. Recently, the generation of spin-entangled electrons via Cooper pair splitting has been theoretically proposed $^{1}$ and experimentally observed. ${ }^{2}$ Nevertheless, one central question is still very challenging: how to detect whether the two electrons are spin-entangled and to what extent they are entangled.

One way to demonstrate the entanglement is Bell tests, ${ }^{3,4}$ which were designed to display the failure of local hidden variable theories. The maximal violation of the Bell inequality can give the concurrence, ${ }^{5}$ which is a measure of the twoparticle entanglement. ${ }^{6}$ Several proposals for Bell tests in solid-state devices have been theoretically suggested. ${ }^{7}$ In order to find the maximal violation caused by two entangled spins, one needs to precisely measure the correlations between two spins with arbitrary polarization directions, which remains a challenge in solid-state experiments.

On the other hand, the quantum eraser consisting of two entangled photons has already been realized experimentally in quantum optics. ${ }^{8}$ In principle, there are two steps to achieve a quantum optics eraser. ${ }^{9}$ First, the which-way information of the signal photon is registered and, according to the complementarity principle, the decoherence of the signal photon occurs. Second, a proper measurement is performed on the entangled partner of the signal photon to erase the which-way information so that the interference recurs.

In this work, we show that two entangled electrons can form a spintronic quantum eraser, and more importantly, the eraser serves as a substitute for the Bell test to quantitatively probe the two-electron entanglement. It is found that the Fano factor exhibits the Aharonov-Bohm (AB) oscillation ${ }^{10}$ with its amplitude being a function of the concurrence. It has also been reported that the tests of either entanglement or the Einstein-Podolsky-Rosen paradox are more robust against the decoherence effects than the Bell tests. ${ }^{11}$ Since the decoherence effects are usually significant in solids, the entanglement detection for two solid spins with our spintronic eraser may be easier to achieve than the Bell tests.

The rest of this paper is organized as follows. The spintronic quantum eraser is presented in Sec. II, the measurement of the concurrence is discussed in Sec. III, and finally, we draw our conclusions in Sec. IV.

\section{SPINTRONIC QUANTUM ERASER}

The proposed quantum eraser is sketched in Fig. 1. The entangler represents a sink of spin-entangled electrons. On the theoretical side, an entangler can be realized by use of superconductors ${ }^{1}$ or quantum dots. ${ }^{12}$ On the experimental side, with a bias under the superconducting gap, the spin-entangled electrons can be efficiently created via Cooper pair splitting. ${ }^{2}$ The two electrons from the entangler are separately injected into two channels along the $x$ direction, labeled by $u$ and $l$, respectively. Each channel consists of only a single transverse mode. We denote the electron in channel $u$ as the idler electron (IE) and that in channel $l$ as the signal electron (SE). A longer decoherence length at low temperatures is beneficial to the observation of the entanglement so that we restrict our discussions at zero temperature for simplicity. The state of two spin-entangled electrons is expressed by

$$
\left|\Psi_{0}\right\rangle=\left[\sqrt{\kappa} a_{l \uparrow}^{\dagger}\left(E_{1}\right) a_{u \downarrow}^{\dagger}\left(E_{2}\right) \mp \sqrt{1-\kappa} a_{l \downarrow}^{\dagger}\left(E_{1}\right) a_{u \uparrow}^{\dagger}\left(E_{2}\right)\right]|0\rangle,
$$

where operator $a_{i \sigma}^{\dagger}(E)$ creates an electron with energy $E$ and spin $\sigma$ in channel $i(i=u, l)$, and satisfies the discrete anticommutation relations $\left\{a_{i \sigma}\left(E_{1}\right), a_{i^{\prime} \sigma^{\prime}}^{\dagger}\left(E_{2}\right)\right\}=\delta_{i i^{\prime}} \delta_{\sigma \sigma^{\prime}} \delta_{E_{1} E_{2}}$. The vacuum state $|0\rangle$ is the filled Fermi sea. The concurrence ${ }^{6}$ of the entangled state Eq. (1) is given by $\mathcal{C}=2 \sqrt{\kappa(1-\kappa)}$. Particularly, $\kappa=0,1(\mathcal{C}=0)$ and $\kappa=1 / 2(\mathcal{C}=1)$ represent a direct-product state and a maximally entangled state, respectively, while other values of $\kappa$ correspond to general entangled states with $0<\mathcal{C}<1$. For maximally entangled states, the minus and plus in Eq. (1) stand for the singlet and triplet states, respectively. Although the states expressed by Eq. (1) only represent a subfamily of the entangled spin states, ${ }^{13}$ they are still rather general to cover the common spin-singlet and spin-triplet entangled states from the practical entanglers such as superconductors and quantum dots.

$\left|\Psi_{0}\right\rangle$ in Eq. (1) is the initial state of two electrons emitted from the entangler. The final state after scattering can be obtained by investigating all the transport processes of the $\mathrm{SE}$ and the IE, respectively. The SE in channel $l$ travels through a 


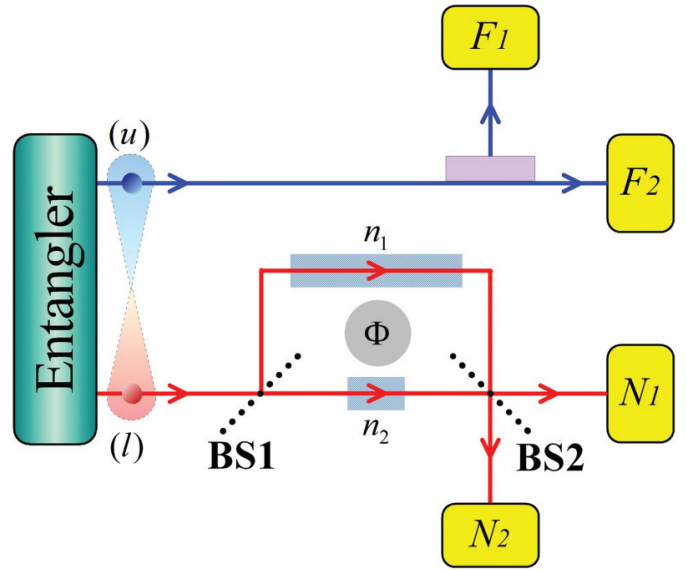

FIG. 1. (Color online) Illustration of a spintronic quantum eraser. Two entangled electrons are injected from the entangler into channels $u$ and $l$. The electron in channel $u$ travels through a spin filter (shaded area) and reaches leads $F_{1}$ and $F_{2}$. The electron in channel $l$ travels through a Mach-Zehnder interferometer and reaches leads $N_{1}$ and $N_{2}$. The Mach-Zehnder interferometer consists of two beam splitters, BS1 and BS2, labeled by dotted lines. The Rashba spin-orbit coupling exists in the shaded regions in paths $n_{1}$ and $n_{2}$, and a magnetic flux $\Phi$ is enclosed by the two paths.

Mach-Zehnder interferometer and finally reaches leads $N_{1}$ and $N_{2}$, respectively. The Mach-Zehnder interferometer, which has already been realized for electrons in experiments, ${ }^{14,15}$ consists of two beam splitters (BS1 and BS2) and a magnetic flux $\Phi$ enclosed by two paths, as shown in Fig. 1.

There is no backscattering when the SE goes through BS1, ${ }^{15,16}$ and the transmission amplitudes into paths $n_{1}$ and $n_{2}$ are assumed to be $t_{1}$ and $t_{2}$, respectively. The wave function of the electrons after BS1 evolves into

$$
\begin{aligned}
\left|\Psi_{1}\right\rangle= & {\left[\sqrt{\kappa}\left(t_{1} a_{n_{1} \uparrow}^{\dagger}+t_{2} a_{n_{2} \uparrow}^{\dagger}\right) a_{u \downarrow}^{\dagger}\right.} \\
& \left.\mp \sqrt{1-\kappa}\left(t_{1} a_{n_{1} \downarrow}^{\dagger}+t_{2} a_{n_{2} \downarrow}^{\dagger}\right) a_{u \uparrow}^{\dagger}\right]|0\rangle,
\end{aligned}
$$

where energy indexes, $E_{1}$ for the $\mathrm{SE}$ and $E_{2}$ for the IE, are omitted for simplicity. In a Mach-Zehnder interferometer with BS1 and BS2, one finds that both spin-up and spin-down SEs travel through two paths simultaneously, leading to an interference pattern determined by the relative phase $\varphi=$ $\operatorname{Arg}\left(t_{1} t_{2}^{*}\right)$. Since the relative phase is controlled by the magnetic flux enclosed by two paths, the interference effect here is the so-called AB oscillation. ${ }^{10}$ The BS1 in solids can be prepared by the quantum point contact in two-dimensional electron gas, and the strongest interference occurs at $\left|t_{1}\right|=\left|t_{2}\right|=1 / \sqrt{2}$, which is realizable in experiments. ${ }^{15,16}$

In our spintronic Mach-Zehnder interferometer shown in Fig. 1, a finite Rashba spin-orbit coupling $\alpha_{R}{ }^{17}$ exists in the Rashba regions in two paths, which substantially alters the interference pattern. The Rashba Hamiltonian for electrons moving one-dimensionally along the $x$ direction is given by $H_{R}=-\alpha_{R} k_{x} \sigma_{y}$, where $k_{x}$ is the wave vector and $\sigma_{y}$ is the Pauli matrix. ${ }^{17}$ The transfer of the SE through the Rashba region can be described by a unitary operator $U=\exp \left(-i \theta_{R} \sigma_{y} / 2\right),{ }^{18}$ implying a spin rotation about the $y$ axis by an angle $\theta_{R}=$ $-2 m^{*} \alpha_{R} L / \hbar^{2}$, where $m^{*}$ is the effective mass of the electron and $L$ is the length of the Rashba region. We set the length of the Rashba region in path $n_{1}$ to be three times of that in path $n_{2}$ so that the spin rotation angles in the two paths satisfy $\theta_{n_{1}}=3 \theta_{n_{2}}$. By tuning gate voltages, $\alpha_{R}$ and therefore the spin rotation angles can be controlled. We adopt here $\theta_{n_{1}}=$ $3 \theta_{n_{2}}=-3 \pi / 2$, which can be achieved in realistic systems. For example, the parameters in InGaAs/InAlAs systems are $m^{*}=0.046 m_{0}$ with $m_{0}$ being the mass of a free electron and $\alpha_{R} \approx 0.39 \times 10^{-11} \mathrm{eV} \cdot \mathrm{m},{ }^{19}$ and thus a spin rotation angle of $-3 \pi / 2$ can be obtained with a Rashba region of the length about $1 \mu \mathrm{m}$.

After the Rashba spin precession, the wave function of the electrons can be derived by applying transfer operator $U$, and is written as

$$
\begin{aligned}
\left|\Psi_{2}\right\rangle= & {\left[\sqrt{\kappa}\left(-t_{1} a_{n_{1} x}^{\dagger}+t_{2} a_{n_{2} \bar{x}}^{\dagger}\right) a_{u \downarrow}^{\dagger}\right.} \\
& \left.\mp \sqrt{1-\kappa}\left(t_{1} a_{n_{1} \bar{x}}^{\dagger}+t_{2} a_{n_{2} x}^{\dagger}\right) a_{u \uparrow}^{\dagger}\right]|0\rangle,
\end{aligned}
$$

where subscripts $x$ and $\bar{x}$ denote the $\operatorname{spin}-x$ and $\operatorname{spin}-\bar{x}$ states, two orthogonal states with spins polarized parallel and antiparallel to the $x$ direction, respectively. One finds that the which-way information of the SE is registered by both electron spins. For example, if the SE is spin- $x$ and the IE is spin-down, the SE must travel in path $n_{1}$. In other words, the traveling paths of the SE are completely distinguishable by use of both electron spins. According to the complementarity principle, the vanishing of the $\mathrm{AB}$ oscillation should be expected. We note that, as shown in Eq. (2), the IE alone can not destroy the interference between two paths. It is the Rashba spin-orbit coupling here that acts as a which-way information detector. Our scheme is a spintronic version of the quantum eraser.

The scattering at BS2 is similar to that at BS1 and can be described by a spin-independent unitary matrix $S=\left(\begin{array}{cc}r & t \\ t^{\prime} & r^{\prime}\end{array}\right)$, where amplitudes $r$ and $t$ correspond to transfers from path $n_{1}$ and path $n_{2}$ to lead $N_{1}$, respectively, and amplitudes $t^{\prime}$ and $r^{\prime}$ correspond to transfers into lead $N_{2}$. By incorporating matrix $S$ into Eq. (3), the electron state after BS2 reads

$$
\begin{aligned}
\left|\Psi_{3}\right\rangle= & {\left[\sqrt { \kappa } \left(-t_{1} r b_{N_{1} x}^{\dagger}-t_{1} t^{\prime} b_{N_{2} x}^{\dagger}+t_{2} t b_{N_{1} \bar{x}}^{\dagger}\right.\right.} \\
& \left.+t_{2} r^{\prime} b_{N_{2} \bar{x}}^{\dagger}\right) a_{u \downarrow}^{\dagger} \mp \sqrt{1-\kappa}\left(t_{1} r b_{N_{1} \bar{x}}^{\dagger}+t_{1} t^{\prime} b_{N_{2} \bar{x}}^{\dagger}\right. \\
& \left.\left.+t_{2} t b_{N_{1} x}^{\dagger}+t_{2} r^{\prime} b_{N_{2} x}^{\dagger}\right) a_{u \uparrow}^{\dagger}\right]|0\rangle,
\end{aligned}
$$

where operator $b_{j \sigma}^{\dagger}$ creates a spin- $\sigma$ electron in lead $j$, representing the outgoing waves after scattering.

In order to find the final state of the two entangled electrons, one should also consider the transportation of the IE, which travels through a spin filter and reaches leads $F_{1}$ and $F_{2}$, respectively. There are no restrictions for electrons going into lead $F_{2}$, but only spin- $y$ electrons (spins polarized along the $y$ direction) can penetrate through the spin filter (shaded region in Fig. 1) and arrive at lead $F_{1}$. In other words, the spin- $\bar{y}$ electrons are excluded from lead $F_{1}$ and can only reach lead $F_{2}$, and the spin- $y$ electrons can reach either leads $F_{1}$ or $F_{2}$. Such a spin filter can be achieved experimentally with a half-metal tunnel junction ${ }^{20}$ or a Zeeman-split quantum dot $^{21}$ attached between channel $u$ and lead $F_{1}$. Both spin filters of half-metal junctions and quantum dots can generate an output current nearly $100 \%$ spin polarized..$^{20,21}$ 
The scattering for the IE at the spin filter can be described by the following relations:

$$
\begin{aligned}
& a_{u \uparrow}^{\dagger}=\frac{1}{\sqrt{2}} \gamma_{1} b_{F_{1}}^{\dagger}+\frac{1}{\sqrt{2}} \gamma_{2} b_{F_{2} y}^{\dagger}+\frac{1}{\sqrt{2}} b_{F_{2} \bar{y}}^{\dagger}, \\
& a_{u \downarrow}^{\dagger}=\frac{-i}{\sqrt{2}} \gamma_{1} b_{F_{1}}^{\dagger}+\frac{-i}{\sqrt{2}} \gamma_{2} b_{F_{2} y}^{\dagger}-\frac{-i}{\sqrt{2}} b_{F_{2} \bar{y}}^{\dagger},
\end{aligned}
$$

where $\gamma_{1,2}$ are amplitudes for spin- $y$ electrons transmitted into leads $F_{1,2}$, respectively. The final state of the two entangled electrons is given by inserting Eq. (5) into Eq. (4).

The current contributed by a pair of entangled electrons emitted from the entangler can be obtained by the standard scattering matrix approach. The current operator in lead $j$ $\left(j=N_{1}, F_{1}\right)$ is given by ${ }^{22}$

$$
I_{j}(t)=\frac{e}{h v} \sum_{E E^{\prime} \sigma} b_{j \sigma}^{\dagger}(E) b_{j \sigma}\left(E^{\prime}\right) \exp \left[i\left(E-E^{\prime}\right) t / \hbar\right],
$$

where $v$ is the density of states at the Fermi level.

The currents in leads $N_{1}$ and $F_{1}$ are obtained by the expectation values of $I_{j}(t)$ in the final state. One finds that $\left\langle I_{F_{1}}\right\rangle=(e / h v)\left(\Gamma_{1} / 2\right)$ and $\left\langle I_{N_{1}}\right\rangle=(e / h v)\left(T_{1} R+T_{2} T\right)$, with $\Gamma_{1}=\left|\gamma_{1}\right|^{2}, T_{1,2}=\left|t_{1,2}\right|^{2}, R=|r|^{2}$, and $T=|t|^{2}$. Both currents are constants determined only by the transmission probabilities. Since there is a finite Rashba spin-orbit coupling in paths $n_{1}$ and $n_{2}$ acting as a which-way information detector, the interference between two paths is completely destroyed. The current in lead $N_{1}$ can be understood in a straightforward manner. The SE arrives at lead $N_{1}$ through two paths. One is to move along path $n_{1}$ and then from path $n_{1}$ to lead $N_{1}$, resulting in a current $T_{1} R$. The other is to move along path $n_{2}$ and then from path $n_{2}$ to lead $N_{1}$, resulting in a current $T_{2} T$. The total current in lead $N_{1}$ is just a sum of these two contributions.

In order to recover the $\mathrm{AB}$ oscillation, the which-way information must be erased by a proper measurement on the IE. If the SE and the IE are not entangled, the measurement on the IE could not recover the interference, and the maximal recovery will occur if two electrons are maximally entangled. It is expected that the amount of the recovered interference will give the information on the measure of entanglement, concurrence.

In quantum optics, the interference recurs with a coincidence counting of the correlated measurements on entangled photons. The counterpart here is the shot noise, a currentcurrent correlation, between leads $N_{1}$ and $F_{1}$. In general, the noise power between leads $j$ and $j^{\prime}$ is given by ${ }^{22}$

$$
S_{j j^{\prime}}(\omega)=\lim _{\tau \rightarrow \infty} \frac{h v}{\tau} \int_{0}^{\tau} d t e^{i \omega t}\left\langle\delta I_{j}(t) \delta I_{j^{\prime}}(0)\right\rangle,
$$

where $\delta I_{j}=I_{j}-\left\langle I_{j}\right\rangle$, and the average is made under the final state. With the help of Eqs. (4)-(6), the averages in Eq. (7) can be obtained. For example, we have

$$
\begin{aligned}
\left\langle I_{N_{1}}(t) I_{F_{1}}(0)\right\rangle= & \left(\frac{e}{h v}\right)^{2}\left\langle\Psi_{f}\right| \sum_{E E^{\prime} \sigma} b_{N_{1} \sigma}^{\dagger}(E) b_{N_{1} \sigma}\left(E^{\prime}\right) \\
& \times e^{i\left(E-E^{\prime}\right) t / \hbar} \sum_{E^{\prime \prime} E^{\prime \prime \prime}} b_{F_{1}}^{\dagger}\left(E^{\prime \prime}\right) b_{F_{1}}\left(E^{\prime \prime \prime}\right)\left|\Psi_{f}\right\rangle,
\end{aligned}
$$

and the final state

$$
\begin{aligned}
\left|\Psi_{f}\right\rangle= & \frac{1}{\sqrt{2}}\left[\sqrt { \kappa } \left(-t_{1} r b_{N_{1} x}^{\dagger}-t_{1} t^{\prime} b_{N_{2} x}^{\dagger}+t_{2} t b_{N_{1} \bar{x}}^{\dagger}\right.\right. \\
& \left.+t_{2} r^{\prime} b_{N_{2} \bar{x}}^{\dagger}\right)\left(-i \gamma_{1} b_{F_{1}}^{\dagger}-i \gamma_{2} b_{F_{2} y}^{\dagger}+i b_{F_{2} \bar{y}}^{\dagger}\right) \\
& \mp \sqrt{1-\kappa}\left(t_{1} r b_{N_{1} \bar{x}}^{\dagger}+t_{1} t^{\prime} b_{N_{2} \bar{x}}^{\dagger}+t_{2} t b_{N_{1} x}^{\dagger}\right. \\
& \left.\left.+t_{2} r^{\prime} b_{N_{2} x}^{\dagger}\right)\left(\gamma_{1} b_{F_{1}}^{\dagger}+\gamma_{2} b_{F_{2} y}^{\dagger}+b_{F_{2} \bar{y}}^{\dagger}\right)\right]|0\rangle .
\end{aligned}
$$

In Eq. (9), the operators for the $\mathrm{SE}\left(b_{N_{1}}^{\dagger}\right.$ and $\left.b_{N_{2}}^{\dagger}\right)$ are of energy $E_{1}$ and those for the IE $\left(b_{F_{1}}^{\dagger}\right.$ and $\left.b_{F_{2}}^{\dagger}\right)$ are of energy $E_{2}$. After utilizing the anticommutation relations of the electron operators and $b_{j \sigma}|0\rangle=0$, one obtains

$$
\begin{aligned}
\left\langle I_{N_{1}}(t) I_{F_{1}}(0)\right\rangle= & \left(\frac{e}{h v}\right)^{2} \frac{\Gamma_{1}}{2}\left[T_{1} R+T_{2} T\right. \\
& \left.\mp 2 \sqrt{\kappa(1-\kappa)}\left(i t_{1} r t_{2}^{*} t^{*}+\text { c.c. }\right)\right] .
\end{aligned}
$$

Inserting Eq. (10) into Eq. (7), the zero-frequency $(\omega=0)$ noise power between leads $N_{1}$ and $F_{1}$ is found to be

$$
S_{N_{1} F_{1}}= \pm \frac{e^{2}}{h v} \mathcal{C} \Gamma_{1} \sqrt{T_{1} T_{2} R T} \sin \varphi,
$$

where the phase difference between the two scattering amplitudes at $\mathrm{BS} 2, \operatorname{Arg}\left(r t^{*}\right)$, is incorporated into the definition of $\varphi \cdot{ }^{23}$ It is seen clearly that the $\mathrm{AB}$ oscillation recurs in the shot noise. Although all the transmission probabilities in Eq. (11) can be obtained beforehand, the strongest interference pattern occurs with $T_{1}=T_{2}=1 / 2$ and $R=T=1 / 2$, which can be experimentally realized for beam splitters. ${ }^{15,16}$ Under this condition, the Fano factor, defined as $F=\left(S_{N_{1} F_{1}} /\left\langle I_{F_{1}}\right\rangle\right) /(e / 2)$, is obtained as

$$
F= \pm \mathcal{C} \sin \varphi
$$

\section{DISCUSSIONS}

There are rich physics contained in Eq. (12). First, the Fano factor is a sinusoidal function of the phase controlled by the external magnetic flux, revealing the recovery of the $\mathrm{AB}$ oscillation and a quantum eraser effect in solids. Second, the two-electron entanglement is quantitatively probed by the Fano factor. The concurrence is just the amplitude of the $\mathrm{AB}$ oscillation. Third, the Fano factor in a spintronic quantum eraser can also distinguish the singlet and triplet states. The different signs in Eq. (12) represent a $\pi$-phase shift in the AB oscillation. For the maximally entangled states $(\mathcal{C}=1)$, the plus and the minus are corresponding to the singlet and triplet states, respectively.

Notably, although Eq. (12) is obtained with an initial state spin polarized along the $z$ direction, the present scenario is still valid in more general cases. For an initial state of $\operatorname{spin} \sigma=\cos \alpha \sigma_{z}+\sin \alpha \sin \beta \sigma_{y}+\sin \alpha \cos \beta \sigma_{x}$, with $\alpha$ and $\beta$ being two arbitrary polarization angles, the noise power reads $S_{N_{1} F_{1}}=\left(e^{2} \Gamma_{1} / 4 h v\right)\left[\mathcal{C}^{2} \sin ^{2} \alpha \sin ^{2} \beta \pm\right.$ $\left.\mathcal{C}\left(\cos ^{2} \beta+\sin ^{2} \beta \cos ^{2} \alpha\right)\right] \sin \varphi$. For $\alpha=\beta=0$, Eq. (11) is recovered. More practically, the entangled electrons emitted from an $s$-wave superconductor are spin unpolarized and the measured noise power is an average over angles $\alpha$ and $\beta$. The average current in lead $F_{1}$ remains unchanged and the 
Fano factor is given by $F=(1 / 3)(1+2 \mathcal{C}) \sin \varphi$. In this case, the Fano factor still shows an $\mathrm{AB}$ oscillation behavior with an amplitude being the concurrence. Considering an experimentally observed $\mathrm{AB}$ oscillation of the Fano factor with an amplitude $A>1 / 3$, the concurrence is obtained as

$$
\mathcal{C}=(3 A-1) / 2 \text {. }
$$

We here wish to pinpoint that the present spintronic quantum eraser is distinctly different from some other schemes of mesoscopic erasers. ${ }^{24}$ Our proposal consists of two entangled electrons and utilizes the spin-orbit coupling as the which-way information detector. Although the variation of the current correlations in a Mach-Zehnder interferometer has been exhibited, the entanglement still needs to be detected by the Bell test. ${ }^{25}$ In this work, the concurrence is quantitatively determined from the $\mathrm{AB}$ oscillation of the Fano factor, which serves as a substitute for the Bell test and paves a direct way for probing quantitatively the two-electron entanglement.

It is also worthwhile to compare the present method for entanglement detection with the Bell test. Both methods can probe the entanglement, however, there are at least two important differences between them. First, they have different working principles. The present method employs an idea of quantum eraser, which is a result of the complementarity principle rather than the Bell's theorem. Second, with respect to quantifying the concurrence of two spin-entangled electrons, the present method is easier to implement than the Bell test. In a Bell test, the concurrence is given by the maximal violation of the Bell inequality. ${ }^{5}$ To achieve this goal, one needs to precisely measure the spin-spin correlations, but a perfect separation and counting of spins along arbitrary polarization directions remains a challenge in experiments. In the present method, the concurrence can be simply determined from the $\mathrm{AB}$ oscillation of the Fano factor, which is a charge current correlation. Although we use a spin filter in channel $u$, a perfect separation and counting of different spins is not required. Since we do not require $\Gamma_{1}=1$, the spin- $y$ and spin- $\bar{y}$ electrons in channel $u$ are not fully separated. The only prerequisite is that the output current in lead $F_{1}$ is fully spin polarized, which is realizable in experiments. ${ }^{20,21}$ However, the electrons flowing into lead $F_{2}$ can be either spin- $y$ or spin- $\bar{y}$ ones.

The main building blocks of the proposed eraser are an electronic Mach-Zehnder interferometer and a spin filter, both of which have already been implemented. ${ }^{15,20,21}$ In addition, the order of magnitude of the decoherence length in metal and semiconductor mesoscopic systems is usually several micrometers at low temperatures. ${ }^{26}$ For GaAs, the spin decoherence length can even exceed $100 \mu \mathrm{m} .{ }^{27}$ Therefore, the spin decoherence length in a mesoscopic system is long enough to realize the proposed device of a scale about $1 \mu \mathrm{m}$. In this sense, our proposal is very likely to be realized experimentally in the near future.

\section{SUMMARY}

In this paper, we have designed a spintronic quantum eraser to quantitatively probe the two-electron entanglement. Two simple steps are sufficient to find the concurrence. First, the gate voltages and subsequently the Rashba spin-orbit coupling are tuned so that the AB oscillation of the current in lead $N_{1}$ is eliminated. Second, the concurrence is evaluated by measuring the $\mathrm{AB}$ oscillation of the Fano factor. Our proposal paves a direct way for probing quantitatively the two-electron entanglement, which may be regarded as a substitute for the corresponding Bell test.

\section{ACKNOWLEDGMENTS}

We would like to thank Y. Yu and Z.-Y. Xue for fruitful discussions. This work was supported by 973 Program (Grants No. 2011CB922100, No. 2011CBA00205, and No. 2009CB929504), by NSFC (Grants No. 11074111, No. 11174125, and No. 11023002), by PAPD of Jiangsu Higher Education Institutions, by NCET, by the Fundamental Research Funds for the Central Universities, by the GRF (HKU7058/11P) and CRF (HKU-8/11G) of the RGC of Hong Kong.

\footnotetext{
*shen@nju.edu.cn

†zwang@hku.hk

${ }^{1}$ G. B. Lesovik, T. Martin, and G. Blatter, Eur. Phys. J. B 24, 287 (2001); P. Recher, E. V. Sukhorukov, and D. Loss, Phys. Rev. B 63, 165314 (2001).

${ }^{2}$ L. Hofstetter, S. Csonka, J. Nygård, and C. Schönenberger, Nature (London) 461, 960 (2009); L. G. Herrmann, F. Portier, P. Roche, A. L. Yeyati, T. Kontos, and C. Strunk, Phys. Rev. Lett. 104, 026801 (2010); J. Wei and V. Chandrasekhar, Nat. Phys. 6, 494 (2010).

${ }^{3}$ J. S. Bell, Rev. Mod. Phys. 38, 447 (1966).

${ }^{4}$ J. F. Clauser, M. A. Horne, A. Shimony, and R. A. Holt, Phys. Rev. Lett. 23, 880 (1969).

${ }^{5}$ N. Gisin, Phys. Lett. A 154, 201 (1991).

${ }^{6}$ S. Hill and W. K. Wootters, Phys. Rev. Lett. 78, 5022 (1997); W. K. Wootters, ibid. 80, 2245 (1998).
}

${ }^{7}$ S. Kawabata, J. Phys. Soc. Jpn. 70, 1210 (2001); P. Samuelsson, E. V. Sukhorukov, and M. Büttiker, Phys. Rev. Lett. 91, 157002 (2003); C. W. J. Beenakker, C. Emary, M. Kindermann, and J. L. van Velsen, ibid. 91, 147901 (2003); N. M. Chtchelkatchev, G. Blatter, G. B. Lesovik, and T. Martin, Phys. Rev. B 66, 161320(R) (2002).

${ }^{8}$ T. J. Herzog, P. G. Kwiat, H. Weinfurter, and A. Zeilinger, Phys. Rev. Lett. 75, 3034 (1995); S. P. Walborn, M. O. Terra Cunha, S. Padua, C. H. Monken, Phys. Rev. A 65, 033818 (2002).

${ }^{9}$ M. O. Scully and K. Drühl, Phys. Rev. A 25, 2208 (1982).

${ }^{10}$ Y. Gefen, Y. Imry, and M. Ya. Azbel, Phys. Rev. Lett. 52, 129 (1984).

${ }^{11}$ H. M. Wiseman, S. J. Jones, and A. C. Doherty, Phys. Rev. Lett. 98, 140402 (2007); E. G. Cavalcanti, P. D. Drummond, H. A. Bachor, and M. D. Reid, Opt. Express 17, 18693 (2009). 
${ }^{12}$ D. Loss and E. V. Sukhorukov, Phys. Rev. Lett. 84, 1035 (2000); W. D. Oliver, F. Yamaguchi, and Y. Yamamoto, ibid. 88, 037901 (2002).

${ }^{13}$ V. Giovannetti, D. Frustaglia, F. Taddei, and R. Fazio, Phys. Rev. B 74, 115315 (2006).

${ }^{14}$ B. Yurke, Phys. Rev. Lett. 56, 1515 (1986).

${ }^{15}$ Y. Ji, Y. Chung, D. Sprinzak, M. Heiblum, D. Mahalu, and H. Shtrikman, Nature (London) 422, 415 (2003).

${ }^{16}$ R. C. Liu, B. Odom, Y. Yamamoto, and S. Tarucha, Nature (London) 391, 263 (1998); M. Henny, S. Oberholzer, C. Strunk, T. Heinzel, K. Ensslin, M. Holland, and C. Schönenberger, Science 284, 296 (1999); W. D. Oliver, J. Kim, R. C. Liu, and Y. Yamamoto, ibid., 284, 299 (1999).

${ }^{17}$ Yu. A. Bychkov and E. I. Rashba, JETP Lett. 39, 78 (1984).

${ }^{18}$ J. C. Egues, G. Burkard, and D. Loss, Phys. Rev. Lett. 89, 176401 (2002).

${ }^{19}$ S. Datta and B. Das, Appl. Phys. Lett. 56, 665 (1990).

${ }^{20}$ J. S. Moodera, R. Meservey, and X. Hao, Phys. Rev. Lett. 70, 853 (1993).
${ }^{21}$ R. Hanson, L. M. K. Vandersypen, L. H. Willems van Beveren, J. M. Elzerman, I. T. Vink, and L. P. Kouwenhoven, Phys. Rev. B 70, 241304(R) (2004).

${ }^{22}$ G. Burkard, D. Loss, and E. V. Sukhorukov, Phys. Rev. B 61, R16303 (2000).

${ }^{23}$ In fact, phase $\varphi$ in Eq. (11) is a sum of the magnetic flux and a reference phase $\varphi_{0}$, which is accumulated when electrons propagate through each path and each interface. The reference phase $\varphi_{0}$ is a parameter of the circuit and can be determined beforehand by measuring the $\mathrm{AB}$ oscillation behavior of the SE.

${ }^{24}$ G. Hackenbroich, B. Rosenow, and H. A. Weidenmüller, Europhys. Lett. 44, 693 (1998); K. Kang, Phys. Rev. B 75, 125326 (2007); J. Dressel, Y. Choi, and A. N. Jordan, ibid. 85, 045320 (2012).

${ }^{25}$ I. Neder, N. Ofek, Y. Chung, M. Heiblum, D. Mahalu, and V. Umansky, Nature (London) 448, 333 (2007); P. Samuelsson, E. V. Sukhorukov, and M. Büttiker, Phys. Rev. Lett. 92, 026805 (2004).

${ }^{26}$ J. J. Lin and J. P. Bird, J. Phys.: Condens. Matter 14, R501 (2002).

${ }^{27}$ J. M. Kikkawa and D. D. Awschalom, Phys. Rev. Lett. 80, 4313 (1998); Nature (London) 397, 139 (1999). 